\title{
Melting behaviour of molecular mixed crystalline materials: measurement with adiabatic calorimetry and modelling using ULTRACAL
}

\author{
Peter R. van der Linde, Mark Bolech, Remco den Besten, \\ Marcel L. Verdonk, J. Cornelis van Miltenburg and \\ Harry A. J. Oonk ${ }^{a}$ \\ Chemical Thermodynamics Group, Debye Institute, Faculty of Chemistry, \\ Utrecht University, Padualaan 8, 3584 CH Utrecht, The Netherlands
}

\begin{abstract}
The melting behaviour of binary mixed crystalline materials was measured with adiabatic calorimetry. The experimental results (heat capacity against temperature graphs) are explained in terms of a model that is based on the assumption that the inhomogeneity of the materials can be described by a Gaussian distribution function. Although this model, called ULTRACAL, has only one adjustable parameter, it is capable of reproducing the experimental data with a good degree of accuracy, as demonstrated for various samples of (1,4-dichlorobenzene +1 ,4-dibromobenzene). ULTRACAL thus provides a link between the adiabatic calorimetry data and the solid-liquid $T x$ phase diagram. It is shown that the model can be used for the determination of phase diagrams. By using ULTRACAL to assess the heat capacity against temperature graphs of five samples, the solid-liquid $T x$ phase diagram of (1,3,5-trichlorobenzene + 1,3,5-tribromobenzene) was determined. (C) 2002 Elsevier Science Ltd. All rights reserved.
\end{abstract}

KEYWORDS: molecular mixed crystals; melting; adiabatic calorimetry; modelling; phase diagram

\section{Introduction}

A mixed crystalline material, in going from a solid to a liquid state, gives rise ideally to the type of heat capacity against temperature graph $\left(c_{p}-T\right)$ shown in figure 1 . The sharp rise at the solidus temperature and sharp fall at the liquidus temperature allow the phase diagram characteristics for a given composition to be determined.

In practice, however, the ideal $c_{p}-T$ graph is hardly ever observed. The extent to which it can be approximated depends upon experimental procedures and particularly upon the degree of homogeneity of the sample, i.e. the way in which the sample was prepared. Because of low solid-state diffusion rates, ${ }^{(1)}$ mixed crystalline samples of molecular substances have to be prepared starting with a fluid phase: melt, vapour or solution in a

${ }^{a}$ To whom correspondence should be addressed (E-mail: h.a.j.oonk@chem.uu.nl). 


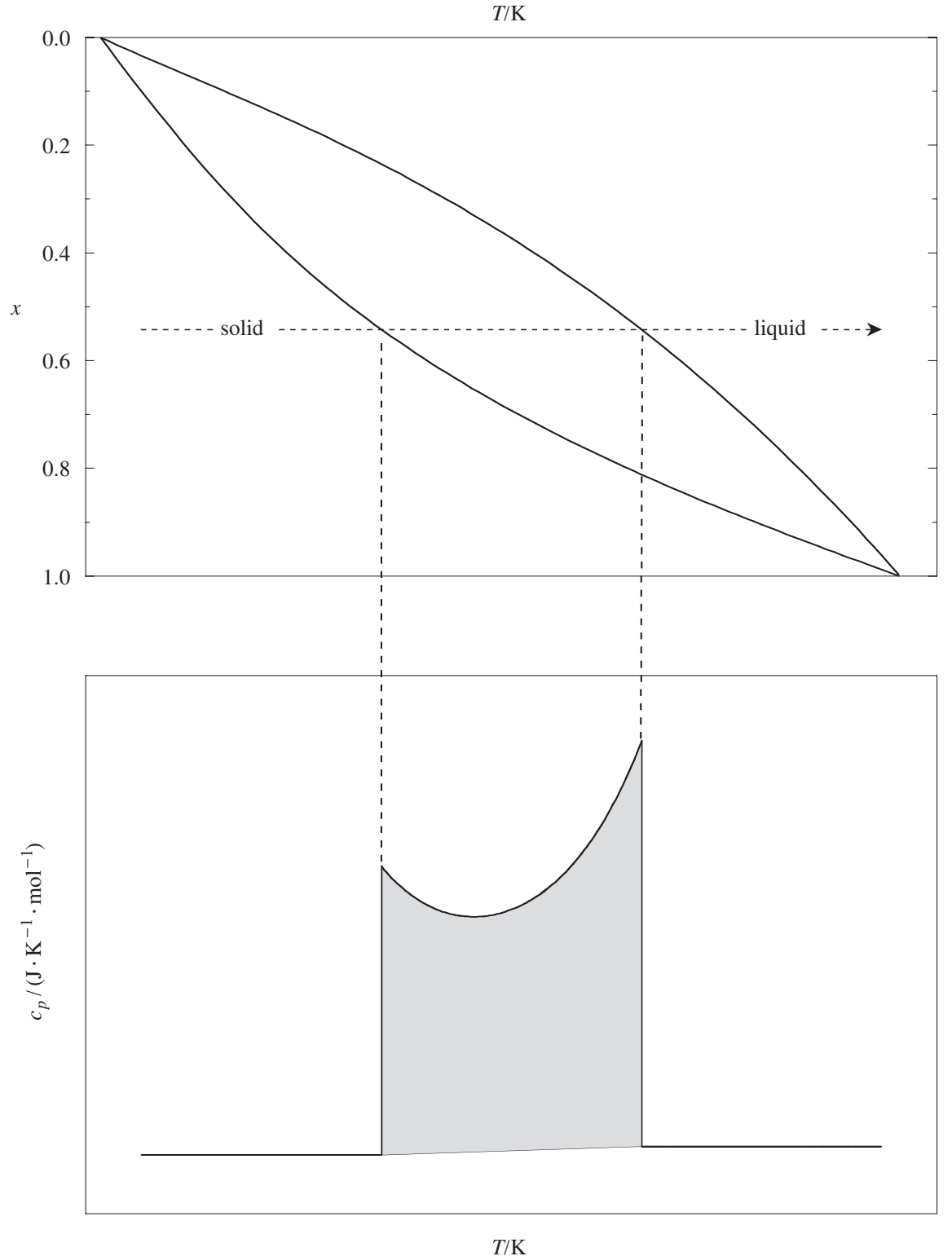

FIGURE 1. Relation between the solid-liquid $T x$ phase diagram of a binary mixture (upper graph) and the theoretical ideal $c_{p}-T$ graph (lower graph). The shaded area in the lower graph corresponds to the heat of melting for the given composition.

solvent. The necessity of using a fluid medium, however, has unavoidable adverse effects on the degree of homogeneity of the material (as, from an equilibrium point of view, 
the transition from one mixed state, the fluid medium, to the mixed crystalline state is accompanied by a change in composition).

In searching for standardisation of sample preparation procedures, our group, particularly in the initial phase of its existence, explored the possibilities of the zone levelling technique. A zone levelling instrument was designed by Kolkert and its performance was tested on (naphthalene $+\beta$-naphthol). ${ }^{(2,3)}$ The method of zone levelling exceeded our expectations. It yielded solidus and liquidus data in good agreement with the results of thermodynamic analysis of phase diagrams and thermochemical data. Similar results were obtained by Bouwstra for (trans-diphenylethene + trans-azobenzene). ${ }^{(4)}$

Zone levelling provided a solid basis for examining the possibilities and limitations of less sophisticated methods of sample preparation such as quenching from a melt or rapid co-precipitation from a solution in a highly volatile solvent. These relatively simple methods allow the preparation of $5 \mathrm{~g}$ samples of mixed crystalline material in about half an hour, against half a month in the case of zone levelling. This paper focuses on the method of rapid co-precipitation from solution and its application to (1,4-dichlorobenzene $+1,4$ dibromobenzene) and (1,3,5-trichlorobenzene + 1,3,5-tribromobenzene) .

In figure 2 three examples of $c_{p}-T$ graphs corresponding to the same mean composition $\left(x_{\mathrm{m}}=0.52\right)$ for $(1,4$-dichorobenzene $+1,4$-dibromobenzene $)$ are given. As can be seen, $c_{p}-T$ graphs may cover a spectrum of shapes: from the ideal graph, the solid curve, when the compositional homogeneity is 100 per cent, to the graph represented by open squares. The latter was obtained using a sample prepared by quenching a liquid mixture. It is clear that the material consists of regions with different compositions covering a large part of the mole fraction range; see also Bouwstra. ${ }^{(4)}$ The full circles in figure 2 represent the experimental results for a sample prepared by co-precipitation from solution. The shape of the curve does suggest that the degree of homogeneity is rather high but, unfortunately, shows also that direct and accurate information on the phase diagram cannot be extracted. Therefore we have decided to explore the use of a method, a mathematical model for describing the inhomogeneity of the sample, for determining accurately solidus and liquidus temperatures without the need for input data other than those on pure substances and mixing properties.

The investigations ${ }^{(5)}$ were carried out as follows. Initially, the known solid-liquid $T x$ phase diagram of (1,4-dichlorobenzene + 1,4-dibromobenzene) was used to test the adopted model. Secondly, the model was taken as the starting point for an iterative procedure by which a limited set of experimental $c_{p}-T$ graphs of $(1,3,5-$ trichlorobenzene + 1,3,5-tribromobenzene) were interpreted and the (unknown) solid-liquid $T x$ phase diagram was established. Lastly, and guided by the outcome of the model calculations, the possibility of determining solidus and liquidus temperatures directly from $c_{p}-T$ graphs, i.e. without using the mathematical procedure, was examined.

\section{Materials}

The substances 1,4-dichlorobenzene, 1,4-dibromobenzene, 1,3,5-trichlorobenzene, and 1,3,5-tribromobenzene were obtained from Aldrich Chemical Co. Inc., Milwaukee, WI, U.S.A. 1,4-Dichlorobenzene and 1,3,5-trichlorobenzene were purified by sublimation 


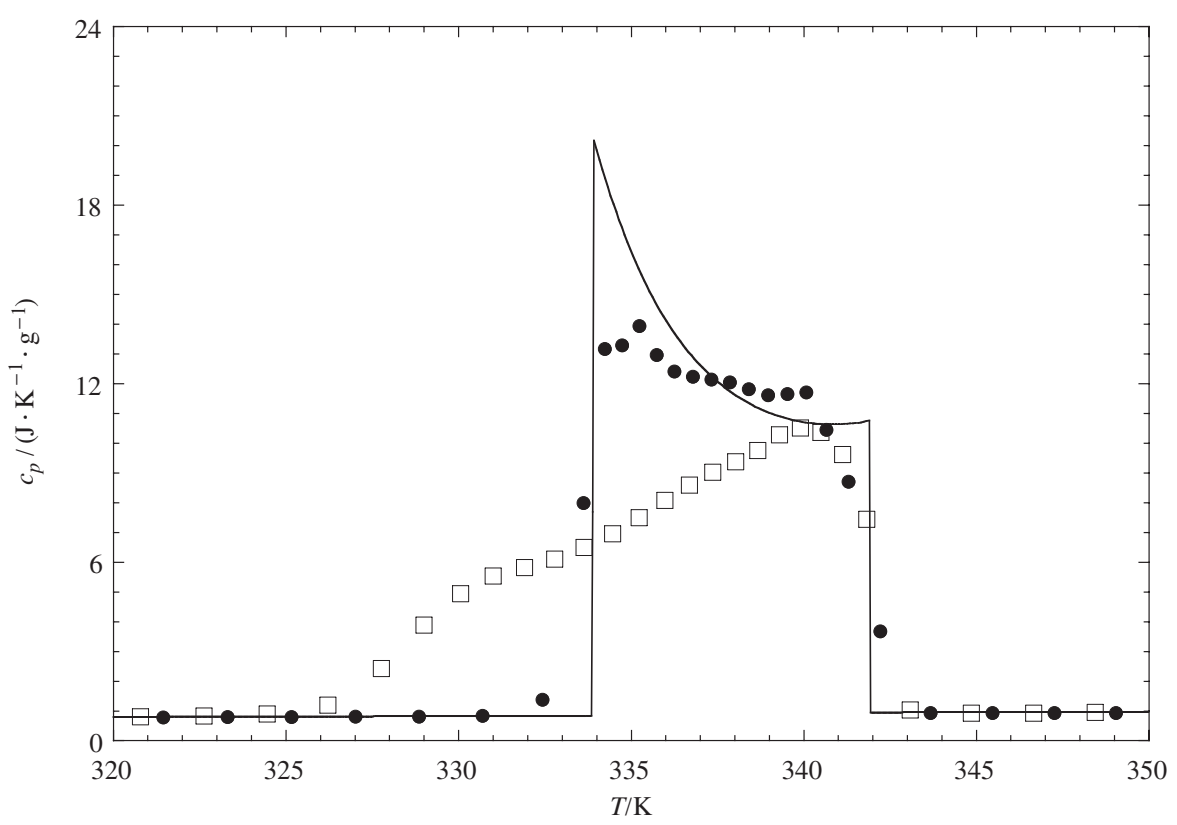

FIGURE 2. Examples of $c_{p}-T$ graphs at the mean composition $x_{\mathrm{m}}=0.52$ for $(1,4-$ dichorobenzene +1 ,4-dibromobenzene): $\bullet$, sample prepared by co-precipitation from solution; $\square$, sample prepared by quenching of a liquid mixture; - , theoretical ideal $c_{p}-T$ graph. Calculations were performed with heat capacity expressed in $\mathrm{J} \cdot \mathrm{K}^{-1} \cdot \mathrm{g}^{-1}$. In this way the error due to conversion of the experimental heat capacity data to $\mathrm{J} \cdot \mathrm{K}^{-1} \cdot \mathrm{mol}^{-1}$ using the average molar mass can be avoided. All data presented in figures 2-6 are available in tabular form upon request. In addition, the computer program ULTRACAL can be obtained from the authors.

in vacuo under continuous evacuation at approximately $1 \mathrm{~Pa}$. For the other substances this purification method did not yield satisfactory results.

1,4-Dibromobenzene (light-brown coloured) was dissolved in ethanol. $200 \mathrm{ml}$ of the saturated solution were filtered and poured into four litres of distilled water. The precipitate was dried at $p=1 \mathrm{~Pa}$. Subsequently, the white substance was sublimated once.

Dark-brown coloured 1,3,5-tribromobenzene was sublimated once. The yellow product was further purified by recrystallization from ethanol. After centrifugation during three minutes at 2400 r.p.m., the substance was isolated and washed with a small amount of acetone. Finally, the product was dried in vacuo.

The purity of the substances was checked by means of adiabatic calorimetry. Except for 1,3,5-tribromobenzene (mole fraction purity $=0.9990$ ), the mole fraction purities were found to be at least 0.9998 .

\section{PREPARATION OF MIXED SAMPLES}

Mixed crystalline materials prepared by rapid co-precipitation from solution were used in the investigations. Pure substances in the desired proportions were dissolved in diethyl 
ether and a mixed solid material was allowed to form through fast evaporation of the solvent, i.e. supersaturation was driven far enough for the whole mass to crystallize at about the same moment. In this way mixed crystalline materials were obtained within $30 \mathrm{~min}$.

\section{Experimental}

All measurements were made using a partially reconstructed and improved version of the adiabatic calorimeter $\mathrm{CAL} \mathrm{V},{ }^{(6)}$ the original construction of which was described extensively by van Miltenburg et al. ${ }^{(7)}$ and van Bommel. ${ }^{(8)}$

Each measurement consisted of successive heating periods and stabilization periods of $500 \mathrm{~s}$ and $3600 \mathrm{~s}$ duration, respectively. The stabilization periods were extended from the normal $1500 \mathrm{~s}$ to $3600 \mathrm{~s}$ in order to come closer to equilibrium.

\section{The ULTRACAL model}

The method of co-precipitation from solution yields a mixed crystalline material that is a fine powder, i.e. a collection of numerous very small crystallites. Furthermore, the product is formed within minutes which suggests a more or less random crystallization process. These two observations form the basis for the following three assumptions that underly the model called ULTRACAL (the name ULTRACAL is derived from Calorimetrica Ultraiectina, Ultraiectum being the Latin name for Utrecht):

(i) each of the numerous crystallites is homogeneous in composition and shows ideal melting behaviour, i.e. in accordance with figure 1; (ii) a given crystallite $i$ has its own composition $x_{i}$, and the distribution of compositions in the sample as a whole is a Gaussian function around the mean composition $\left(x_{\mathrm{m}}\right)$; this distribution function is characterized by a standard deviation $\sigma$, referred to here as "dispersion"; (iii) there is no interaction between the crystallites, i.e. each crystallite melts independently and there is no mixing between the formed liquid phases.

Consequently, the $c_{p}-T$ graph of the sample as a whole is the weighted sum of numerous ideal $c_{p}-T$ graphs. Now let $k$ represent the number of different compositions in the sample and $M_{i}$ the ideal $c_{p}-T$ graph corresponding to composition $x_{i}$, the $c_{p}-T$ graph of the sample $\left(M_{\mathrm{T}}\right)$ can then be given as:

$$
M_{\mathrm{T}}=Z^{-1} \cdot \sum_{i=1}^{k} \exp \left\{-\left(x_{i}-x_{\mathrm{m}}\right)^{2} / 2 \cdot \sigma^{2}\right\} \cdot M_{i},
$$

where $Z$, the normalization constant, is given by

$$
Z=\sum_{j=1}^{k} \exp \left\{-\left(x_{j}-x_{\mathrm{m}}\right)^{2} / 2 \cdot \sigma^{2}\right\} .
$$

Using the pure substance data (table 1), the optimized phase diagram, and the excess enthalpy of melting (table 3), ULTRACAL calculations were carried out on $c_{p}-T$ graphs of 
TABLE 1. Molar thermodynamic properties of the pure substances 1,4-dichlorobenzene $\left(\mathrm{diCl} ; M=147.00 \mathrm{~g} \cdot \mathrm{mol}^{-1}\right), 1,4$-dibromobenzene $\left(\mathrm{diBr} ; M=235.91 \mathrm{~g} \cdot \mathrm{mol}^{-1}\right), 1,3,5-$ trichlorobenzene (triCl; $M=181.45 \mathrm{~g} \cdot \mathrm{mol}^{-1}$ ), and 1,3,5-tribromobenzene (triBr; $M=$ $\left.314.80 \mathrm{~g} \cdot \mathrm{mol}^{-1}\right)$. Heat capacity data $c_{p}^{\mathrm{o}}$ are given at the reference temperature $\theta=298.15 \mathrm{~K}$

$$
\left(p^{\mathrm{o}}=10^{5} \mathrm{~Pa} ; R=8.314472 \mathrm{~J} \cdot \mathrm{K}^{-1} \cdot \mathrm{mol}^{-1}\right)^{(5)}
$$

\begin{tabular}{|c|c|c|c|c|c|c|}
\hline Substance & $\frac{C_{p, \mathrm{~m}}^{\mathrm{o}}(\mathrm{cr}, \theta)}{R}$ & $\frac{\left(\partial C_{p, \mathrm{~m}}^{\mathrm{o}} / \partial T\right)(\mathrm{cr})}{R \cdot \mathrm{K}^{-1}}$ & $\frac{T_{\mathrm{cr}-1}}{\mathrm{~K}}$ & $\frac{\Delta_{\mathrm{cr}}^{1} H_{\mathrm{m}}^{\mathrm{O}}\left(T_{\mathrm{cr}-1}\right)}{R \cdot \mathrm{K}}$ & $\frac{C_{p, \mathrm{~m}}^{\mathrm{o}}(1, \theta)}{R}$ & $\frac{\left(\partial C_{p, \mathrm{~m}}^{\mathrm{o}} / \partial T\right)(1)}{R \cdot \mathrm{K}^{-1}}$ \\
\hline $\mathrm{diCl}^{a}$ & 17.293 & $5.326 \cdot 10^{-2}$ & 326.05 & 2168.2 & 20.368 & $2.838 \cdot 10^{-2}$ \\
\hline $\mathrm{diBr}$ & 17.480 & $5.297 \cdot 10^{-2}$ & 360.48 & 2452.0 & 21.282 & $2.601 \cdot 10^{-2}$ \\
\hline triCl & 19.042 & $4.637 \cdot 10^{-2}$ & 335.92 & 2111.7 & 22.761 & $2.716 \cdot 10^{-2}$ \\
\hline triBr & 19.432 & $4.002 \cdot 10^{-2}$ & 394.95 & 2612.4 & 23.097 & $3.578 \cdot 10^{-2}$ \\
\hline
\end{tabular}

${ }^{a}$ All data for crystalline 1,4-dichlorobenzene apply to the $\alpha$-phase; the $\alpha$-phase is stable up to $T=$ $306 \mathrm{~K}$, see text.

TABLE 2. Solidus and liquidus temperatures $\left(T^{\text {sol }}\right.$ and $\left.T^{\text {liq }}\right)$ of the optimized $T x$ phase diagrams of $(1,4-$ dichlorobenzene $+1,4$-dibromobenzene) and (1,3,5trichlorobenzene $+1,3,5$-tribromobenzene)

\begin{tabular}{|c|c|c|c|c|c|}
\hline \multicolumn{3}{|c|}{$\begin{array}{l}\text { (1,4-dichlorobenzene }+ \\
\text { 1,3,5-tribromobenzene) }\end{array}$} & \multicolumn{3}{|c|}{$\begin{array}{c}\text { 1,3,5-trichlorobenzene + } \\
\text { 1,4-dibromobenzene }\end{array}$} \\
\hline$x$ & $T^{\mathrm{sol}} / \mathrm{K}$ & $T^{\mathrm{liq} / \mathrm{K}}$ & $x$ & $T^{\mathrm{sol}} / \mathrm{K}$ & $T^{\mathrm{liq} / \mathrm{K}}$ \\
\hline 10 & 326.63 & 326.87 & 0.10 & 337.91 & 340.02 \\
\hline .20 & 327.70 & 329.09 & 0.20 & 340.18 & 346.86 \\
\hline .30 & 329.16 & 332.65 & 0.30 & 342.71 & 354.86 \\
\hline 0.40 & 331.03 & 336.85 & 0.40 & 345.66 & 362.46 \\
\hline .50 & 33 & 3 & 0.50 & 24 & 369.28 \\
\hline 0.60 & 336.50 & 345.46 & 0.60 & 353.82 & 375.34 \\
\hline .70 & 340.48 & 349.52 & 0.70 & 359.93 & 380.81 \\
\hline .80 & 345.62 & 353.3 & 0.80 & 368.30 & 385.82 \\
\hline 0.90 & 352.20 & 357.30 & 0.90 & 3879.84 & 390.49 \\
\hline
\end{tabular}

six different samples. The outcome of the calculations is given in table 4. Typical examples of calculated $c_{p}-T$ graphs along with experimental data are shown in figure 4 .

In practice the compositions are assumed to range from $\left(x_{\mathrm{m}}-5 \cdot \sigma\right)$ to $\left(x_{\mathrm{m}}+5 \cdot \sigma\right)$, and the number of different compositions $k$ is about 500. For calculating an ideal curve $M_{i}$ the molar thermodynamic properties of the pure substances (molar heat capacities of the crystalline and liquid phases, temperature and molar enthalpy of melting), the molar excess enthalpy of melting and the solid-liquid $T x$ phase diagram (solidus and liquidus temperatures as a function of composition) are required as input. 


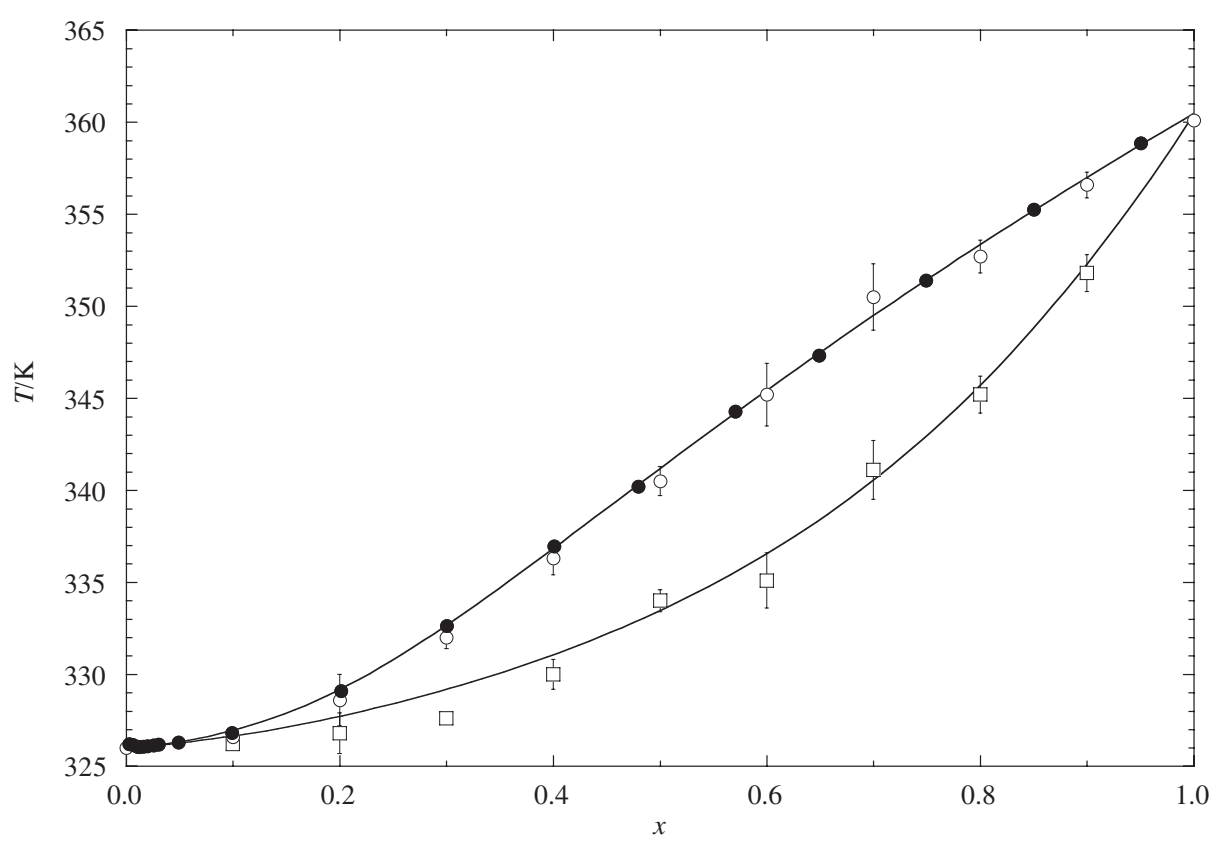

FIGURE 3. Solid $(\alpha)$-liquid Tx phase diagram of (1,4-dichorobenzene + 1,4-dibromobenzene): -, liquidus data by Campbell and Prodan; ${ }^{(9)} \circ$, liquidus data by Haget et al.; ${ }^{(13)} \square$, solidus data by Haget et al.; (13) _ excess Gibbs energy functions of equation type (3): $\left(g_{0}^{1} / \mathrm{J} \cdot \mathrm{mol}^{-1}\right)=0,\left(g_{1}^{1} / \mathrm{J} \cdot \mathrm{mol}^{-1}\right)=0$, $\left(g_{0}^{\mathrm{s}} / \mathrm{J} \cdot \mathrm{mol}^{-1}\right)=1430$, and $\left(g_{1}^{\mathrm{s}} / \mathrm{J} \cdot \mathrm{mol}^{-1}\right)=349$ and the data listed in table 1 (in the calculations the temperature dependence of the transition heat capacity of the pure substances was not taken into account).

TABLE 3. Molar excess enthalpy difference between the mixed crystalline state and mixed liquid state $\Delta_{\mathrm{S}}^{1} H^{\mathrm{E}}$ of $(1,4$-dichlorobenzene + 1,4-dibromobenzene $\}$ and (1,3,5-trichlorobenzene $+1,3,5$ tribromobenzene) derived from experimental $c_{p}-T$ graphs of six samples expressed in terms of the parameters $h_{0}$ and $h_{1}$ as $\Delta_{\mathrm{S}}^{1} H^{\mathrm{E}}(x)=x \cdot(1-x) \cdot\left\{h_{0}+h_{1} \cdot(1-2 \cdot x)\right\}$

\begin{tabular}{|c|c|c|}
\hline System & $\frac{\mathrm{h}_{0}}{\mathrm{~J} \cdot \mathrm{mol}^{-1}}$ & $\frac{\mathrm{h}_{1}}{\mathrm{~J} \cdot \mathrm{mol}^{-1}}$ \\
\hline (1,4-dichlorobenzene $+1,4$-dibromobenzene) & -1983 & -346 \\
\hline (1,3,5-trichlorobenzene $+1,3,5$-tribromobenzene) & -5709 & 1233 \\
\hline
\end{tabular}

In modelling with ULTRACAL the dispersion $\sigma$ is obtained by varying its value until a good agreement between the experimental and calculated $c_{p}-T$ graphs is visually obtained. Although this procedure is not entirely free from subjectivity, the dispersion can be determined with adequate accuracy (the shape of the calculated graph is sensitive to 
TABLE 4. Dispersion $\sigma$ of six samples of $(1,4-$ dichlorobenzene $+1,4$-dibromobenzene) and five samples of (1,3,5-trichlorobenzene $+1,3,5$ tribromobenzene). Apart from the samples with mean composition $x_{\mathrm{m}}=0.60$, which were prepared by zone levelling, all samples were prepared by co-precipitation from solution

\begin{tabular}{|c|c|c|c|}
\hline \multicolumn{2}{|c|}{$\begin{array}{c}\text { (1,4-dichlorobenzene }+ \\
\text { 1,4-dibromobenzene })\end{array}$} & \multicolumn{2}{|c|}{$\begin{array}{c}\text { (1,3,5-trichlorobenzene }+ \\
\text { 1,3,5-tribromobenzene) }\end{array}$} \\
\hline$x_{\mathrm{m}}$ & $\sigma$ & $x_{\mathrm{m}}$ & $\sigma$ \\
\hline 0.21 & 0.032 & 0.20 & 0.017 \\
\hline 0.52 & 0.037 & 0.40 & 0.024 \\
\hline 0.60 & 0.020 & 0.50 & 0.027 \\
\hline 0.60 & 0.021 & 0.68 & 0.020 \\
\hline 0.81 & 0.020 & 0.81 & 0.017 \\
\hline 0.83 & 0.020 & & \\
\hline
\end{tabular}

changes in $\sigma$ of the order \pm 0.001$)$. It may turn out, however, that it is impossible to obtain a good agreement on both the rising and falling edges of the graphs. In such a case, the rising edge of the experimental data was chosen as the target to match, since the third assumption of independently melting crystallites, i.e. no liquid-liquid mixing, is more realistic at the beginning than at the end of the melting process. For a detailed account of the ULTRACAL model and the calculation of ideal $c_{p}-T$ graphs the reader is referred to van der Linde. ${ }^{(5)}$

\section{Results}

\section{(1,4-DICHLOROBENZENE + 1,4-DIBROMOBENZENE)}

As mentioned above, the solid-liquid $T x$ phase diagram is part of the input to ULTRACAL. In the case of (1,4-dichlorobenzene +1 ,4-dibromobenzene) the liquidus, determined by Campbell and Prodan in 1948, ${ }^{(9)}$ is accurately known. As previously demonstrated, ${ }^{(10)}$ Campbell and Prodan's liquidus data are very accurate, but unfortunately their solidus data are not (the data have a systematic error, a common feature of solidus temperatures read from cooling curves). Therefore the solidus was obtained by calculation, using the LIQFIT method. $(4,11,12)$

LIQFIT is an iterative procedure for phase-diagram calculations, in which the liquidus is made to pass through a set of experimental liquidus points. Apart from the experimental liquidus data, the input for LIQFIT consists of the thermodynamic properties of the pure substances and the excess Gibbs energy of either the mixed crystalline state $\left(G^{\mathrm{E}, \mathrm{s}}\right)$ or the liquid state $\left(G^{\mathrm{E}, 1}\right)$. As a rule, and in view of the relatively small temperature range of the solid-liquid region, the change of these excess Gibbs energies with temperature is neglected. The output of LIQFIT consists of (i) the calculated solidus, and (ii) the unknown 
excess function. The excess functions are expressed in terms of the parameters ( $g_{0}$ and $g_{1}$ ) as

$$
G^{\mathrm{E}}(x)=x \cdot(1-x) \cdot\left\{g_{0}+g_{1} \cdot(1-2 \cdot x)\right\} .
$$

The thermodynamic properties of the pure substances used for LIQFIT are given in table 1. Crystalline 1,4-dibromobenzene is isomorphous with the $\alpha$-form of 1,4dichlorobenzene (monoclinic). At temperatures above $306 \mathrm{~K}$ the stable form of 1,4dichlorobenzene is triclinic $(\beta)$, which has a melting point $0.19 \mathrm{~K}$ higher than the metastable melting point of the $\alpha$-form. In the context of this work, however, the existence of the $\beta$-form and its small single-phase field can be ignored.

Measurements of the excess properties of this system ${ }^{(5)}$ have shown that, within the experimental uncertainties, the mixed liquid state can be treated as an ideal mixture. Accordingly, and in terms of equation (3), the computations were made with $g_{0}^{1}=0$ and $g_{1}^{1}=0$ as the parameters of the known function, and $g_{0}^{\mathrm{s}}$ and $g_{1}^{\mathrm{s}}$ as the parameters to be adjusted. The phase diagram obtained by LIQFIT, using Campbell and Prodan's liquidus data as input, is depicted in figure 3 , along with the input data and the solidus and liquidus data of Haget et al. ${ }^{(13)}$ The latter were determined by means of differential thermal analysis d.t.a. using samples prepared by co-precipitation from solution (the liquidus temperatures determined by Haget et al. are systematically $0.35 \mathrm{~K}$ lower than the data of Campbell and Prodan). In table 2 calculated solidus and liquidus temperatures are given for mole fraction steps of 0.1 from $x=0.0$ to $x=1.0$. The calculated excess Gibbs energy of the mixed crystalline state, $G^{\mathrm{E}, \mathrm{s}}$, according to equation (3), is $g_{0}^{\mathrm{s}}=1430 \mathrm{~J} \cdot \mathrm{mol}^{-1}$ and $g_{1}^{\mathrm{s}}=349 \mathrm{~J} \cdot \mathrm{mol}^{-1}$.

\section{(1,3,5-TRICHLOROBENZENE + 1,3,5-TRIBROMOBENZENE)}

In the case of (1,3,5-trichlorobenzene $+1,3,5$-tribromobenzene) the objective was to determine the unknown solid-liquid $T x$ phase diagram, using ULTRACAL for modelling the available experimental $c_{p}-T$ data.

For this system we have used $c_{p}-T$ graphs of just five samples, in combination with the thermodynamic properties of the pure substances (table 1) and the excess enthalpy of melting (table 3). Guided by the ULTRACAL results obtained for (1,4-dichlorobenzene + 1,4dibromobenzene), the experimental $c_{p}-T$ graphs were used to make a first estimate for the solid-liquid $T x$ phase diagram: liquidus and solidus temperatures were read from the graphs and used to construct a complete phase diagram. In order to obtain a thermodynamically correct phase diagram, we have used TXFIT, ${ }^{(14)}$ a computational procedure for the direct derivation of excess properties from experimental phase diagram data. When both the liquidus and the solidus are known and used as input to TXFIT, $G^{\mathrm{E} \text {, s }}$ as well as $G^{\mathrm{E}, 1}$ can be calculated. Hence, there are four adjustable parameters: $g_{0}^{1}, g_{1}^{1}, g_{0}^{\mathrm{s}}$, and $g_{1}^{\mathrm{s}}$, i.e. the parameters of the two excess functions of equation type (3). The output phase diagram of TXFIT is subsequently used as input for ULTRACAL. After minimization of the difference between the experimental and the calculated $c_{p}-T$ graphs (by varying the dispersion for each of the calculated graphs), the experimental and calculated $c_{p}-T$ graphs are compared, and the deviations used to correct the solidus and liquidus temperatures. Subsequently a new iteration is started. 
TABLE 5. Temperatures of inflexion points at rising and falling edges of $c_{p}-T$ graphs, denoted by $T_{i}^{\text {sol }}$ and $T_{i}^{\text {liq }}$, respectively. The solidus and liquidus temperatures that correspond to the optimized phase diagram of (1,4-dichlorobenzene + 1,4-dibromobenzene) and the ULTRACAL phase diagram of (1,3,5-trichlorobenzene $+1,3,5-$ tribromobenzene) are given for comparison; these temperatures were calculated for the mean composition $x_{\mathrm{m}}$ of the sample and are denoted by $T_{\text {calc }}^{\text {sol }}$ and $T_{\text {calc }}^{\text {liq }}$, respectively. The inflexion point temperatures fall into two categories that are distinguished by roman and italic printing

\begin{tabular}{|c|c|c|c|c|}
\hline$x_{\mathrm{m}}$ & $T_{i}^{\mathrm{sol}} / \mathrm{K}$ & $T_{\text {calc }}^{\mathrm{sol}} / \mathrm{K}$ & $T_{i}^{\mathrm{liq}} / \mathrm{K}$ & $T_{\text {calc }}^{\text {liq }} / \mathrm{K}$ \\
\hline \multicolumn{5}{|c|}{ (1,4-dichlorobenzene + 1,4-dibromobenzene) } \\
\hline 0.21 & 327.77 & 327.83 & 329.09 & 329.40 \\
\hline 0.52 & 333.58 & 333.95 & 341.74 & 342.02 \\
\hline 0.59 & 336.59 & 336.33 & 344.95 & 345.25 \\
\hline 0.60 & 336.46 & 336.50 & 345.55 & 345.46 \\
\hline 0.81 & 346.54 & 346.45 & 353.90 & 353.90 \\
\hline 0.83 & 346.98 & 347.30 & 354.45 & 354.42 \\
\hline \multicolumn{5}{|c|}{ (1,3,5-trichlorobenzene $+1,3,5$-tribromobenzene $)$} \\
\hline 0.20 & 340.10 & 340.18 & 347.46 & 346.86 \\
\hline 0.40 & 345.46 & 345.66 & 364.21 & 362.46 \\
\hline 0.50 & 349.40 & 349.24 & 369.35 & 369.28 \\
\hline 0.68 & 358.58 & 358.55 & 379.15 & 379.76 \\
\hline 0.81 & 369.55 & 368.27 & 385.90 & 386.30 \\
\hline
\end{tabular}

Four iterations were sufficient to obtain a good match between the experimental and calculated $c_{p}-T$ graphs. The results consist of (i) calculated $c_{p}-T$ graphs (figure 5), (ii) obtained dispersions (table 4), (iii) calculated phase diagram (figure 6 and table 2), and (iv) values of the adjusted excess Gibbs energy parameters: $g_{0}^{1}=424 \mathrm{~J} \cdot \mathrm{mol}^{-1}$, $g_{1}^{1}=-142 \mathrm{~J} \cdot \mathrm{mol}^{-1}, g_{0}^{\mathrm{s}}=1980 \mathrm{~J} \cdot \mathrm{mol}^{-1}$, and $g_{1}^{\mathrm{s}}=254 \mathrm{~J} \cdot \mathrm{mol}^{-1}$.

\section{DETERMINATION OF PHASE DIAGRAMS WITHOUT ULTRACAL}

Inspection of the results presented in figures 4 and 5 reveals that the ideal $c_{p}-T$ graphs and the experimental data generally intersect at the points where the rising and falling edges of the experimental graphs have their maximum slopes. Taking this into account, and given the fact that an ideal $c_{p}-T$ graph has infinite (that is to say, maximum) slopes at the solidus and liquidus temperatures of the phase diagram, it seems realistic to assume that 


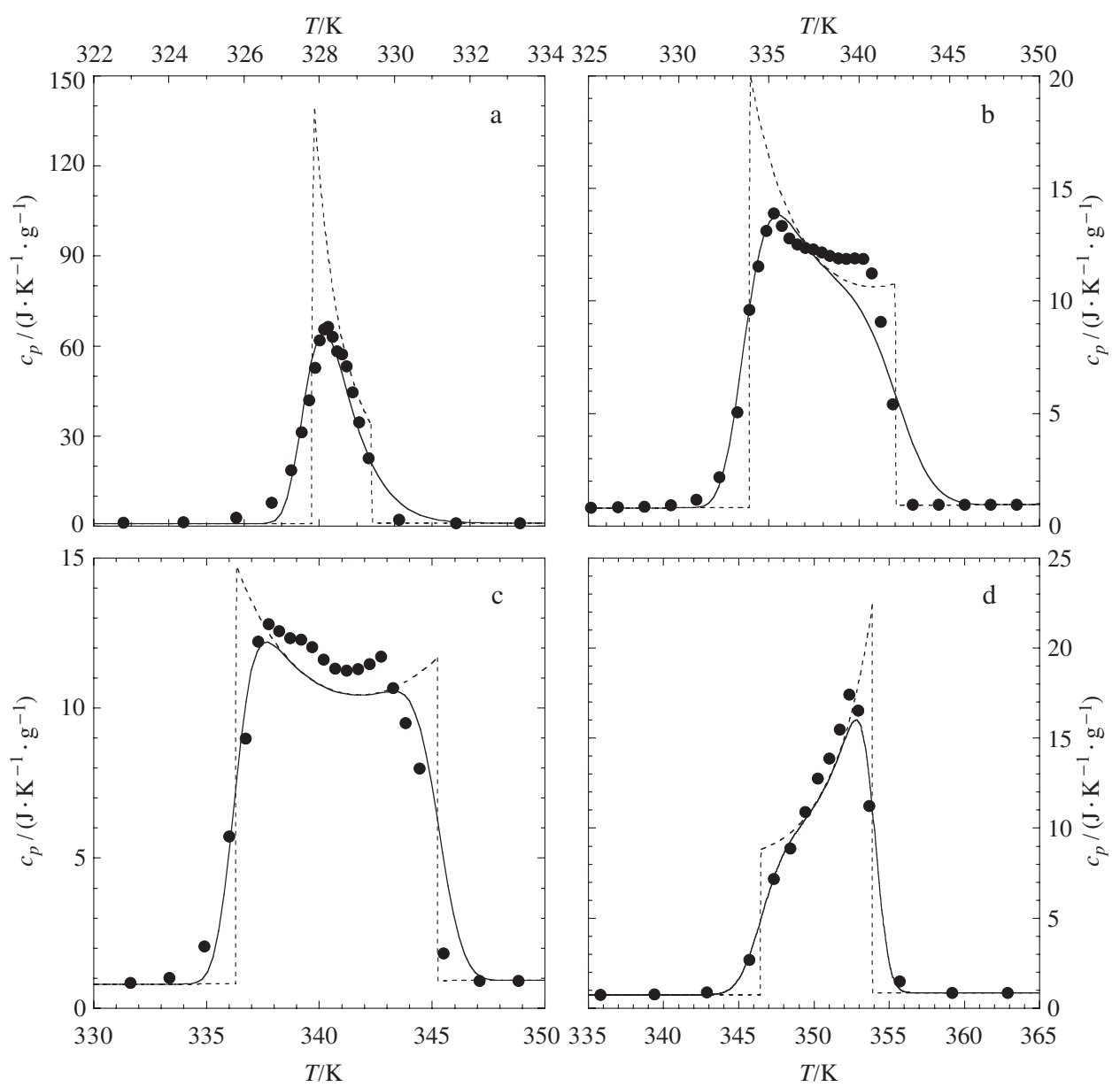

FIGURE 4. $c_{p}-T$ graphs of four samples with mean compositions $x_{\mathrm{m}}=0.21$ (a), $x_{\mathrm{m}}=0.52$ (b), $x_{\mathrm{m}}=0.60(\mathrm{c})$, and $x_{\mathrm{m}}=0.81$ (d) of (1,4-dichlorobenzene +1 ,4-dibromobenzene). Apart from the sample with $x_{\mathrm{m}}=0.60$, which was prepared by zone levelling, all samples were prepared by co-precipitation from solution: $\bullet$, experimental data; ----, ideal $c_{p}-T$ graph;,$- c_{p}-T$ graph calculated by means of ULTRACAL. See table 4 for corresponding dispersions $(\sigma)$. Calculations were performed with heat capacity expressed in $\mathrm{J} \cdot \mathrm{K}^{-1} \cdot \mathrm{g}^{-1}$. In this way the error due to conversion of the experimental heat capacity data to $\mathrm{J} \cdot \mathrm{K}^{-1} \cdot \mathrm{mol}^{-1}$ using the average molar mass can be avoided.

the maximum slopes, at the edges of the $c_{p}-T$ graph of a real sample, will be observed for the solidus and liquidus temperatures of the mean composition. Mathematically, maximum slopes correspond to points of inflexion and for this reason it is worthwhile finding out how close the inflexion point temperatures (read form the $c_{p}-T$ graphs) are to the real solidus and liquidus temperatures. 

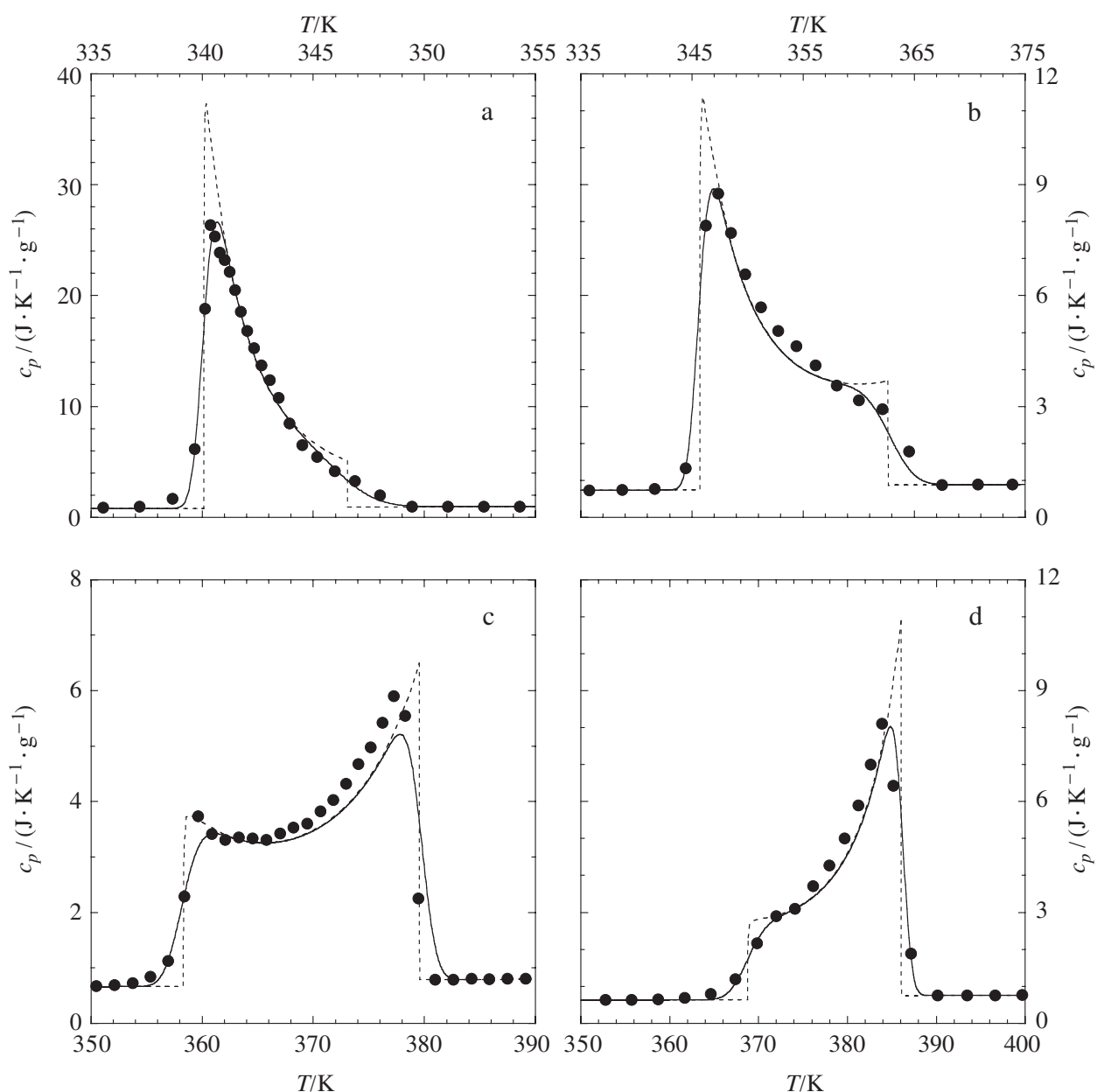

FIGURE 5. $c_{p}-T$ graphs of four samples with mean compositions $x_{\mathrm{m}}=0.20$ (a), $x_{\mathrm{m}}=0.40$ (b), $x_{\mathrm{m}}=0.68(\mathrm{c})$, and $x_{\mathrm{m}}=0.81(\mathrm{~d})$ of (1,3,5-trichlorobenzene $+1,3,5$-tribromobenzene). All samples were prepared by co-precipitation from solution: $\bullet$, experimental data; ----, ideal $c_{p}-T$ graph;

- $c_{p}-T$ graph calculated by means of ULTRACAL. See table 4 for corresponding dispersions $(\sigma)$. Calculations were performed with heat capacity expressed in $\mathrm{J} \cdot \mathrm{K}^{-1} \cdot \mathrm{g}^{-1}$. In this way the error due to conversion of the experimental heat capacity data to $\mathrm{J} \cdot \mathrm{K}^{-1} \cdot \mathrm{mol}^{-1}$ using the average molar mass can be avoided.

The inflexion point temperatures at the rising and falling edges were derived geometrically from the experimental $c_{p}-T$ graphs. The results are given in table 5, where the solidus and liquidus temperatures derived from the calculated phase diagrams are noted for comparison. 


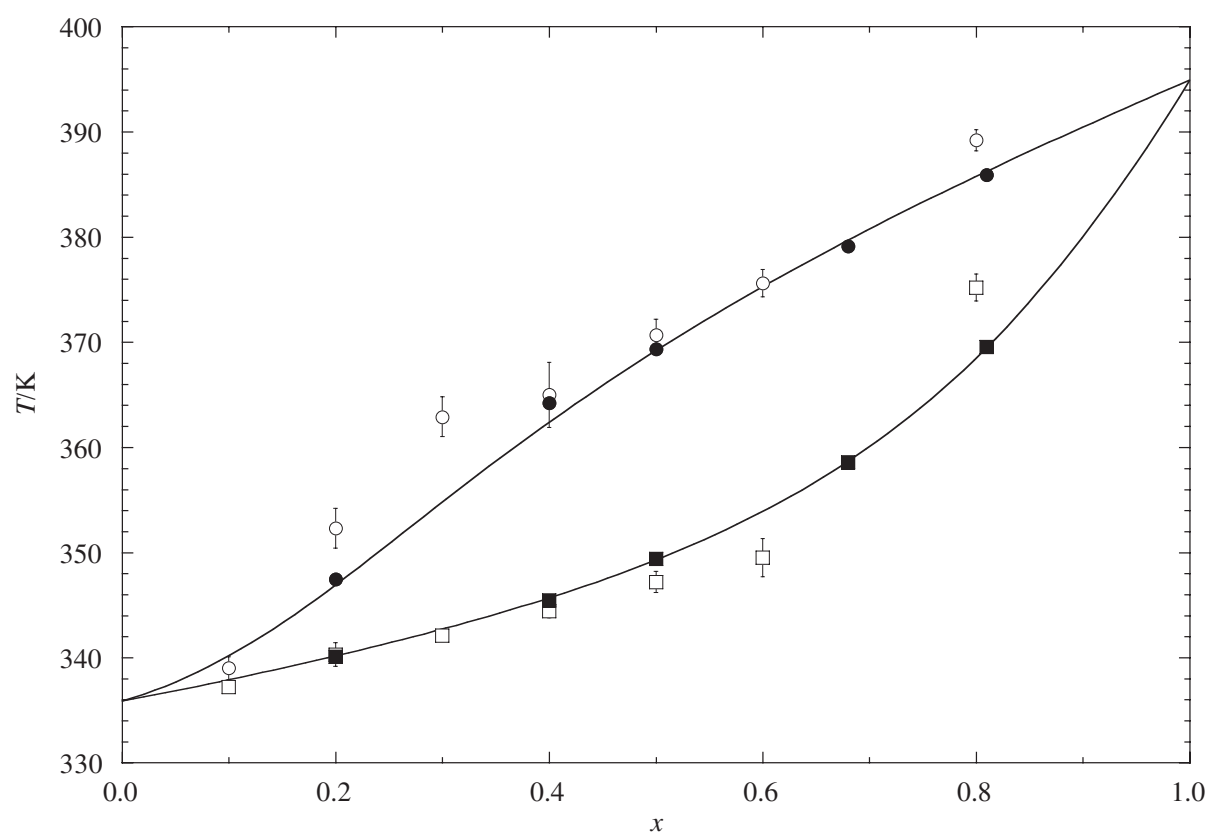

FIGURE 6. Solid-liquid $T x$ phase diagram of (1,3,5-trichorobenzene + 1,3,5-tribromobenzene): -, inflexion point at the falling edge of the $c_{p}-T$ graph; $\mathbf{\square}$, inflexion point at the rising edge of the $c_{p}-T$ graph; $\bigcirc$, liquidus data by Maïga; ${ }^{(15)} \square$, solidus data by Maïga; ${ }^{(15)} \_$, phase diagram calculated using excess Gibbs-energy functions in terms of the parameters of equation (3): $\left(g_{0}^{1} / \mathrm{J} \cdot \mathrm{mol}^{-1}\right)=424,\left(g_{1}^{1} / \mathrm{J} \cdot \mathrm{mol}^{-1}\right)=-142,\left(g_{0}^{\mathrm{s}} / \mathrm{J} \cdot \mathrm{mol}^{-1}\right)=1980$, and $\left(g_{1}^{\mathrm{s}} / \mathrm{J} \cdot \mathrm{mol}^{-1}\right)=254$ and the data listed in table 1 (in the calculations the temperature dependence of the transition heat capacity of the pure substances was not taken into account).

\section{Discussion}

\section{THE ULTRACAL MODEL}

According to figures 4 and 5 , the reproduction by ULTRACAL of the experimental $c_{p^{-}}$ $T$ data, although not complete, is good. Considering the obvious limitations of the underlying model, in particular the fact that there is only one adjustable parameter $(\sigma)$, the results are more than satisfactory. This indicates that the basic assumptions are quite realistic. In other words, the model provides evidence on the existence of a Gaussian distribution of compositions and on the independent melting of the crystallites (at least in the initial stage). However, evidence is no proof and we limit ourselves therefore to the observation that $\sigma$, a measure for the degree of inhomogeneity, should approach the value of zero for mole fractions approaching $x=0.0$ or $x=1.0$. As can be seen in figure 7, our results basically satisfy this a priori condition.

From a comparison of figures 4 and 5 it follows that the $c_{p}-T$ graphs for the unknown phase diagram of (1,3,5-trichlorobenzene $+1,3,5$-tribromobenzene) are equally well 


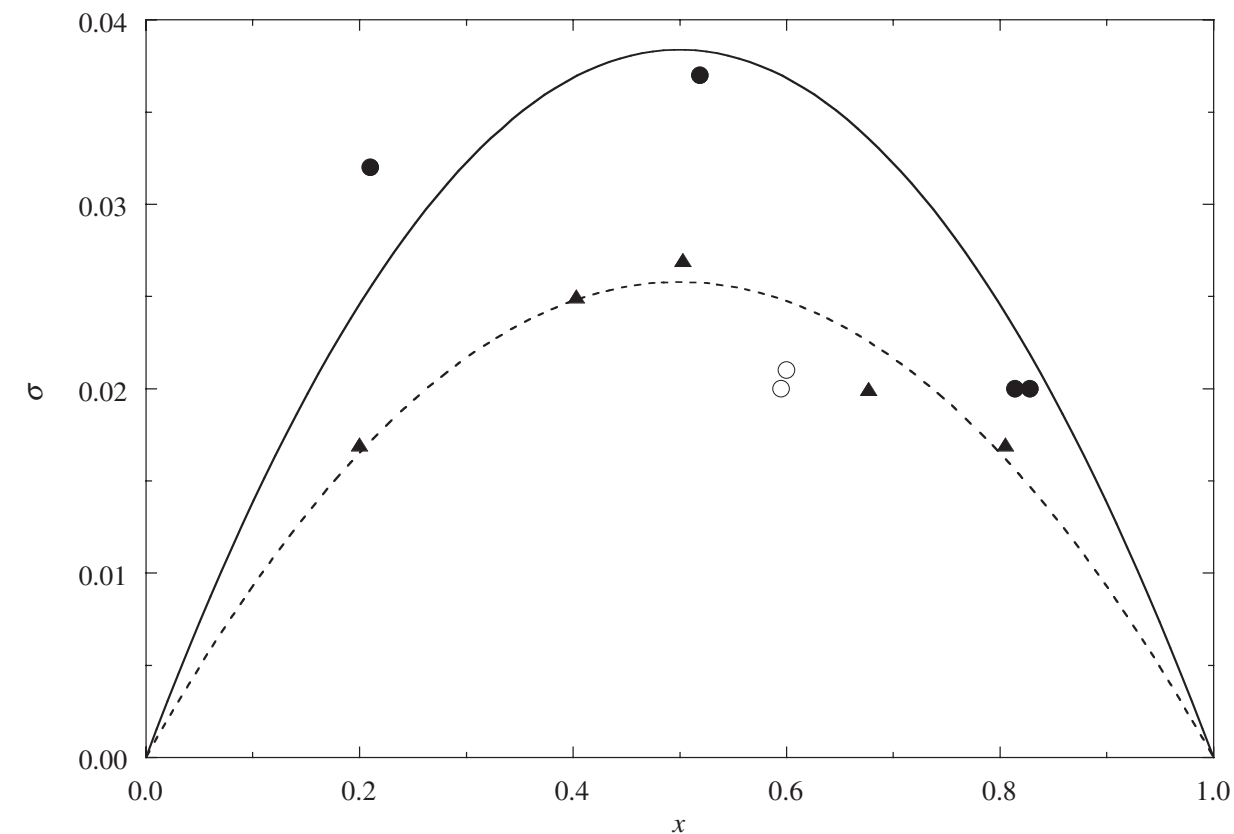

FIGURE 7. Plot of dispersion $\sigma$ as a function of the mean composition $x_{\mathrm{m}}$ of the samples: - (1,4-dichorobenzene + 1,4-dibromobenzene) samples prepared by co-precipitation from solution; O, (1,4-dichorobenzene + 1,4-dibromobenzene) samples prepared by zone levelling; $\boldsymbol{\Delta},(1,3,5-$ trichorobenzene + 1,3,5-tribromobenzene) samples prepared by co-precipitation from solution; - (1,4-dichorobenzene + 1,4-dibromobenzene) fit of the dispersion for the samples prepared by co-precipitation from solution: $\sigma(x)=(0.153 \pm 0.012) \cdot x \cdot(1-x)$; - - - , $(1,3,5$-trichlorobenzene $+1,3,5$-tribromobenzene) fit of the dispersion: $\sigma(x)=(0.103 \pm 0.003) \cdot x \cdot(1-x)$.

reproduced as those of (1,4-dichlorobenzene +1 ,4-dibromobenzene) for which the phase diagram was known and used as input in the computational procedure. It must be noted that the determination of the dispersion and simultaneous determination of the solidus and liquidus temperatures may show some interdependence.

\section{THE INFLEXION POINT TEMPERATURES}

In table 5 two categories of inflexion point temperatures for (1,3,5-trichlorobenzene $+1,3,5$ tribromobenzene) can be distinguished. The three temperatures in italics correspond to edges of the $c_{p}-T$ graphs, such as the falling edge in figures 5(a) and 5(b), where the inflexion point is not clearly discernible. The temperatures of the other category correspond to edges in the $c_{p}-T$ graph where the slope is steep and as a result the point of inflexion can be determined with precision, that is to say within $\pm 0.05 \mathrm{~K}$. In the latter case the absolute average deviation between the calculated and inflexion point temperatures is just $0.22 \mathrm{~K}$. On the basis of this result it can be concluded that, within the experimental errors, the inflexion point temperatures coincide with the solidus and 
liquidus temperatures of the phase diagram. The fact that the deviations are larger for the inflexion point temperatures in italics does not contradict this conclusion.

In the case of (1,4-dichlorobenzene $+1,4$-dibromobenzene) the situation is different: the phase diagram used to make the comparison is fully independent of the experimental $c_{p}-T$ graphs. It means that any differences between the phase diagram and inflexion point temperatures will also reflect a disagreement between the original phase diagram and the phase diagram that would have been obtained if the same procedure applied to $(1,3,5-$ trichlorobenzene + 1,3,5-tribromobenzene) was used. In other words, the differences between inflexion point and calculated temperatures, as displayed in table 5, may also have, apart from one random, a systematic component. As for the latter, both the solidus and liquidus inflexion point temperatures are, on the average, about $0.10 \mathrm{~K}$ lower than the temperatures of the calculated diagram. If a correction of $-0.10 \mathrm{~K}$ is applied, the absolute average deviation between the inflexion point temperatures and the phase diagram reduces to $0.10 \mathrm{~K}$.

\section{ACCURACY AND PRECISION OF SOLIDUS AND LIQUIDUS TEMPERATURES}

In experimental phase diagram research on molecular mixed crystals the work by Campbell and Prodan ${ }^{(9)}$ on (1,4-dichlorobenzene +1 ,4-bromochlorobenzene +1 -bromo4-chlorobenzene) and its binary subsystems constitutes a major contribution. By means of a thermodynamic phase diagram analysis it can be shown ${ }^{(10)}$ that their liquidus temperatures have a high precision (absence of random errors), the absolute average deviation being $0.10 \mathrm{~K}$. From the mutual consistency of the two sets of experimental liquidus temperatures, see figure 3, it follows that the accuracy (absence of systematic errors) of Campbell and Prodan's liquidus points is also high. The systematic error is estimated to be $\pm 0.10 \mathrm{~K}$ at most.

Prior to the present investigation, our opinion was that the $0.10 \mathrm{~K}$-level for liquidus temperatures and a fortiori for solidus temperatures could only be reached by adiabatic calorimetry in combination with zone levelling for the preparation of samples. At the end of the investigation we have arrived at the conclusion that practically the same level can be reached when zone levelling is replaced by rapid co-precipitation from solution.

\section{PHASE DIAGRAM OF (1,3,5-TRICHLOROBENZENE + 1,3,5-TRIBROMOBENZENE)}

In figure 6 the solid-liquid $T x$ phase diagram of (1,3,5-trichlorobenzene + 1,3,5tribromobenzene) determined using ULTRACAL is shown along with the data of Maïga. ${ }^{(15)}$ These data, determined by means of d.t.a., show a rather erratic path which may be explained by limitations of the experimental technique.

Our phase diagram shows the existence of an uninterrupted series of mixed crystals. Maiga, ${ }^{(15)}$ however, concluded that there would be a solid state miscibility gap manifested by a phase diagram with a peritectic three-phase equilibrium. For this reason it is worthwhile having a closer look at the mixing properties. Restricting ourselves to the equimolar composition, the following data can be derived from the results obtained in this work: $\left\{\Delta_{\mathrm{S}}^{1} H^{\mathrm{E}}(x=0.5)\right\}=-1427 \mathrm{~J} \cdot \operatorname{mol}^{-1}$ (see table 3$)$ and $\left\{G^{\mathrm{E}, \mathrm{s}}(x=0.5)\right\}=$ $495 \mathrm{~J} \cdot \mathrm{mol}^{-1}$. Assuming that (i) the molar excess enthalpy difference can be attributed 
to the solid state, and (ii) the excess properties apply to the mean temperature of the solid-liquid two-phase region, $(T / \mathrm{K}) \approx 360$, it can be estimated that the top of the region of demixing is below $(T / K) \approx 225$. At any rate, the conclusion that at room temperature miscibility in the solid state will be complete is justified. Moreover, from a crystallographic point of view complete miscibility is expected in view of the elevated value of the coefficient of crystalline isomorphism $\left(\varepsilon_{\mathrm{m}}=0.91\right){ }^{(15)}$

\section{Conclusions}

In this study mixed crystalline samples were prepared by the method of rapid coprecipitation from an evaporating solvent. For these samples the solid to liquid transition was measured by means of adiabatic calorimetry and the resulting $c_{p}-T$ graphs were analysed by means of the ULTRACAL model.

On the basis of the evidence presented in this work, the following conclusions can be drawn: (i) the ULTRACAL model is appropriate for the simulation of experimental $c_{p}-$ $T$ graphs obtained by adiabatic calorimetry, (ii) the ULTRACAL model can be used to determine the solid-liquid $T x$ phase diagram from a limited set of $c_{p}-T$ graphs, and (iii) the inflexion points on the rising and falling edges of experimental $c_{p}-T$ graphs are virtually at the solidus and liquidus temperatures of the solid-liquid $T x$ phase diagram.

We thank Dr Imogen Poole for critically reviewing the English and Mrs. Hanneke de Vries for preparing the manuscript.

\section{REFERENCES}

1. Wright, J. D. Molecular Crystals: 2nd edition. Cambridge University Press: Cambridge. 1995.

2. Kolkert, W. J. J. Cryst. Growth 1975, 30, 213-219.

3. Kolkert, W. J. Growth of homogeneous organic mixed crystals by repeated pass zone leveling, Thesis, Utrecht University, 1974.

4. Bouwstra, J. A. Thermodynamic and structural investigations of binary systems. Thesis, Utrecht University, 1985.

5. van der Linde, P. R. Molecular mixed crystals from a thermodynamic point of view. Thesis, Utrecht University, 1992.

6. van Miltenburg, J. C.; van Genderen, A. C. G.; van den Berg, G. J. K. Thermochim. Acta 1998, $319,151-162$.

7. van Miltenburg, J. C.; van den Berg, G. J. K.; van Bommel, M. J. J. Chem. Thermodynamics 1987, 19, 1129-1137.

8. van Bommel, M. J. Thermodynamic behaviour of methyl esters of long chain linear carboxylic acids. Thesis, Utrecht University, 1986.

9. Campbell, A. N.; Prodan, L. A. J. Am. Chem. Soc. 1948, 70, 553-561.

10. van Genderen, A. C. G.; de Kruif, C. G.; Oonk, H. A. J. Z. Phys. Chem. Neue Folge 1977, 107, 167-173.

11. Bouwstra, J. A.; Brouwer, N.; van Genderen, A. C. G.; Oonk, H. A. J. Thermochim. Acta 1980, $38,97-107$.

12. Bouwstra, J. A.; Oonk, H. A. J. Calphad 1982, 6, 11-24.

13. Haget, Y.; Housty, J. R.; Maïga, A.; Bonpunt, L.; Chanh, N. B.; Cuevas, M.; Estop, E. J. Chim. Phys. 1984, 81, 197-206.

14. Jacobs, M. H. G. TXFIT, a computer program for the derivation of excess properties of twophase equilibria. Chemical Thermodynamics Group, Utrecht University, 1989. 
15. Maïga, A. Y. Détermination de divers types de diagrammes de phase organiques: mesures isothermiques et mesures isopléthiques. Thesis, University of Bordeaux I, 1983.

(Received 26 July 2000; in final form 12 October 2001)

WA $00 / 040$ 